\title{
Direct and Indirect P-glycoprotein transfers in MCF7 breast cancer cells
}

\author{
JANET DYSON \\ Mathematical Institute, University of Oxford, United Kingdom \\ FRANK LE FOLL \\ Laboratory of Ecotoxicology, University of Le Havre, 76058 Le Havre, France \\ Pierre Magal and Ahmed Noussair \\ Univ. Bordeaux, IMB, UMR 5251, F-33400 Talence, France \\ CNRS, IMB, UMR 5251, F-33400 Talence, France. \\ JENNIFER PASQUIER \\ Stem Cell and Microenvironment Laboratory, Weill Cornell Medical College \\ in Qatar, Education City, Qatar Foundation, Doha PO: 24144, Qatar \\ Department of Genetic Medicine, Weill Cornell Medical College, New York, NY, USA
}

November 4, 2018

\begin{abstract}
The efflux protein P-glycoprotein ( $P$-gp) is over expressed in many cancer cells and has a known capacity to confer multi-drug resistance to cytotoxic therapies. We provide a mathematical model for the direct cellto-cell transfer of proteins between cells and the indirect transfer between cells and the surrounding liquid. After a mathematical analysis of the model, we construct an adapted numerical scheme and give some numerical simulations. We observe that we obtain a better fit with the experimental data when we take into account the indirect transfer of the protein released in a dish. This quantity, usually neglected by the experimenters, seems to influence the results.
\end{abstract}

Key words. Indirect transfers, kinetic, quasi-linear equation, population dynamics models

\section{Introduction}

Multi-drug resistance (MDR) is a phenomenon by which tumor cells exhibit resistance to a variety of chemically unrelated chemotherapeutic drugs. The classical form of multi-drug resistance is connected to overexpression of membrane P-glycoprotein (P-gp), which acts as an energy dependent drug efflux 
pump. The $\mathrm{P}$-gp is the major overexpressed resistance protein in many cancer cells and has a known capacity to confer multi-drug resistance to cytotoxic therapies (see $[1,2,12,15,16,17]$ for further references on the biological properties of P-gp). P-gp expression is known to be controlled by genetic and epigenetic mechanisms.

In 2005 and thereafter, however, it has been discovered that sensitive cells can become resistant by an additional mechanism involving a non-genomic acquisition of functional P-glycoproteins transmitted by donor cells (see [1, 9, 13]). Of course this physical transfer of a protein that retains intrinsic chemotherapeutical resistance is of major significance for diagnosis and treatment design of cancer. The consequences of cell to cell (direct) P-gp transfers have been considered by Hinow et al [6] and Pasquier et al [12]. In these works, the transfer is modeled by an integro-partial differential equation of Boltzmann type.

More recently new experiments have been performed to understand more precisely the different modalities of non-genomic P-gp transfers (see Pasquier et al. [13]). It has been observed that cell to cell P-gp transfers can occur first by direct contact between cells. This process occurs locally in space, and uses Tunneling nanoTubes ( $\mathrm{TnTs}$ ) which build membrane bridges between cells to transfer P-gp. This first way of transferring P-gp is called here direct transfer (or local transfer).

In [13], a second way of transferring P-gp has been observed. Actually, cells overexpressing $\mathrm{P}$-gp can release a fraction of their P-gp packed into the membrane of extracellular vesicles $(\mathrm{EVs})$ shed within the tumoral environment. The EVs diffuse in liquids and eventually can be recaptured by cells, including drug-sensitive ones, transferring their P-gp content into the docking plasma membranes. The experimental evidence for this process is summarized in the Figures 1 and 2.

In Figure 1, EVs were prepared by purification from the liquid culture medium of P-gp overexpressing cells, a doxorubicin-resistant human breast cancer cell line MCF-7/DOXO, by a two-step ultracentrifugation procedure [13]. EVs were then tagged with a green fluorescent probe incorporated in their membrane. Cultured drug-sensitive variants of MCF-7 cells were stained in violet and exposed to the purified green fluorescent EVs for incubation times extending from 0 to 6 bhours. Cells were imaged by using confocal microscopy. Micrographs show a time-dependent increase of peripheral green fluorescence in violet MCF-7, corresponding to a progessive capture of EVs. 

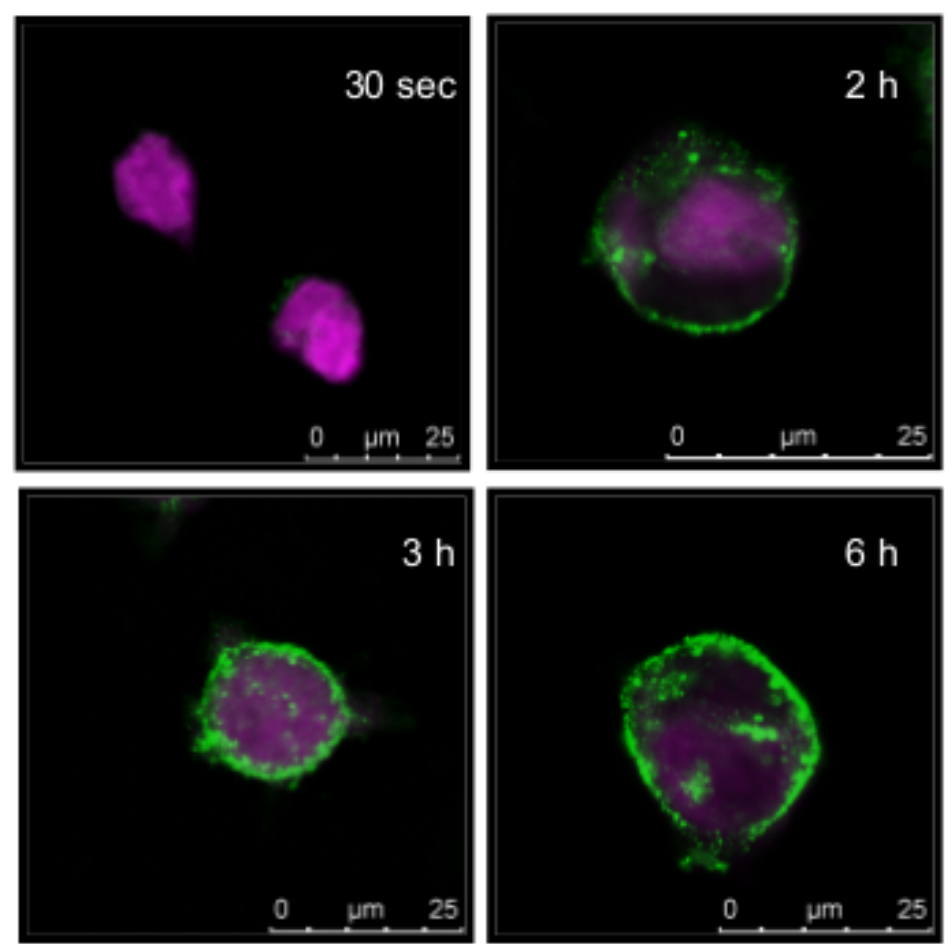

Figure 1: The above figure is taken from [13]. In this experiment $E V$ s were extracted and purified from a culture of P-gp overexpressing human breast cancer MCF-7 cells. The microparticles were labeled with a green fluorescent probe that specifically stains cell membranes. Sensitive non P-gp expressing MCF-7 cells tagged in violet were exposed to the green-labeled microparticles and imaged by confocal microcopy. Micrographs a), b), c) and d) correspond the MCF-7 cells observed respectively after 30s, 2h, 3h and 6h of exposure to microparticles, respectively.

In Figure 2, as in Figure 1, drug sensitive MCF-7 cells were incubated with EVs purified from the culture medium of P-gp overexpressing cells. Sensitive MCF-7 cells were analyzed by flow cytometry after membrane staining with a fluorescent antibody (PE-UIC2) directed against P-gp (in A) and also according to their ability to efflux a fluorescent substrate of P-gp (in C). Analyses were carried out after 0 to $72 \mathrm{~h}$ of incubation with EVs. Corresponding fluorescence histograms are superimposed, showing a progressive shift to the right (in A) or to the left (in $\mathrm{C}$ ) towards the region corresponding to increased levels of P-gp (in A) or decreased levels of intracellular P-gp substrate due to intensified efflux activity (in C). Histograms B and D depict quantitative levels of P-gp (in B) and intracellular content of P-gp substrate (in D) from the above described analyses over time. From those experiments, a transient acquisition of a func- 
tional amount of efflux pump can be established. The peak of P-gp transfer, corresponding to the peak of efflux activity, is observed after 12 hours of incubation with purified EVs. A gradual degradation of EVs and/or proteins can be accounted for by the subsequent vanishing of P-gp in transferred sensitive MCF-7.

EVs are released by all cells and relevant for both physiological and pathological processes. However, EVs have been shown to be shed in greater amounts by tumour than from normal cells (see D'Souza-Schorey and Clancy [4]). Interestingly, Lopez-Rodrigues et al. [10] have shown that EVs from tumoral cell lines contain a high level of P-gp. Moreover, in some circumstances, chemotherapy induces an increased release of EVs from cancer cells (see Samuel et al. [16]). Therefore, EVs-mediated P-gp transfers may quantitatively weigh on MDR dissemination into tumours but remain poorly investigated. 
A

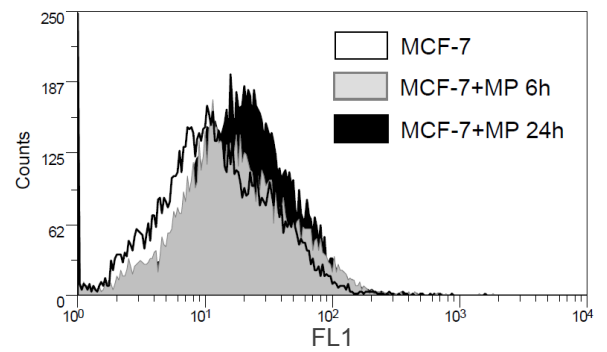

B

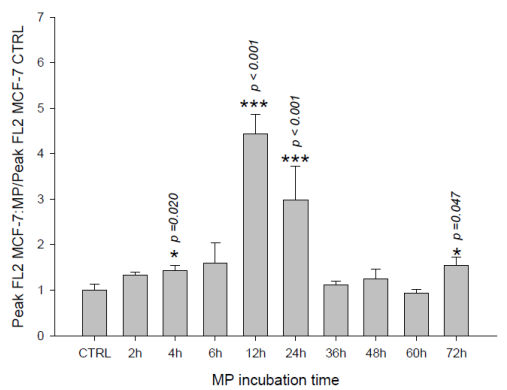

C

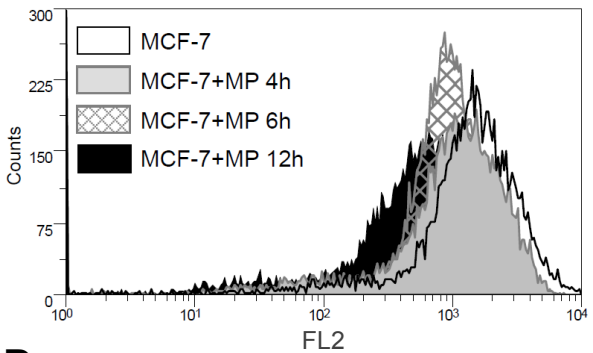

D

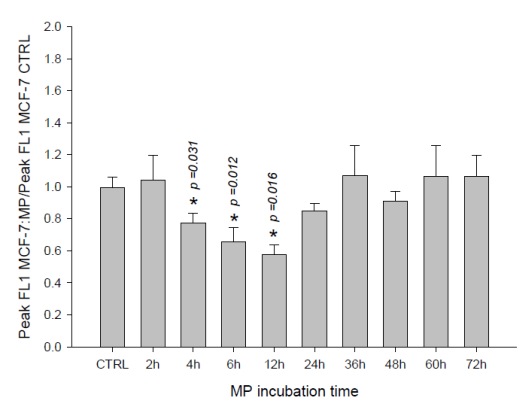

Figure 2: $\quad$ The above figure is taken from [11]. MCF-7 cells exposed to EVs as in Figure 1 were analyzed by flow cytometry to monitor quantitatively their P-gp content and efflux activity over time. A, membrane P-gp content in MCF-7 treated for 6 to 24 hours with EVs extracted from multidrug resistant MCF-7. B, relative increase of membrane P-gp content in MCF-7 treated with MPs extracted from MCF- $\ \backslash D O X O$ was transient, significant after 4 hours and peaked after 12 hours of exposure. Results are expressed as mean SEM of $n=2-10$ replicates. C, P-gp activity in MCF-7 treated for 4 to 12 hours with MPs extracted from multidrug resistant MCF-7. Efflux activity is measured by the decrease of intracellular accumulation of a fluorescent P-gp susbtrate. $D$, relative increase of $P$-gp activity, expressed as a drop of intracellular fluorescence, in MCF-7 treated with MPs extracted from MCF- $\ \backslash D O X O$ was transient, significant after 4 hours and peaked after 12 hours of exposure. Results are expressed as mean \pm SEM of $n=2-10$ replicates. Results significantly different from control are indicated $\left({ }^{*} p<0.05,{ }^{* *} p<0.01,{ }^{* * *} p<0.001\right)$.

The goal of this article is to investigate the influence of EVs-mediated P- 
gp transfers, herein called indirect or remote transfers, onto the overall MDR dynamics in a tumour cell population.

The first aim is to build and to analyze a model describing indirect transfers. Such a class of partial differential equations models appears to be new, and the main challenge will be to combine both direct and indirect transfers. Our goal in this article is to build a mathematical model which can reproduce the Figure $2 \mathrm{~A}$ and C. In Section 6, we obtain some figures which are comparable to the Figure $2 \mathrm{~A}$ and $\mathrm{C}$. Therefore Figure 2 is serving as a concrete motivation for our paper.

The main difficulty in the article will be to study the well posedness of the model in which both the direct and indirect transfers are involved. These problems present considerable mathematical challenges. As far as we know such a class of nonlinear transport operators has not been considered before. The plan of the paper is the following. In section 2 we consider separately models of direct transfers and of indirect transfers. In section 3 we construct the group of solutions of a related transport equation by relating it to the transport equation on the real line. In section 4 we define weak and mild solutions of the full model combining both direct and indirect transfers, and give a sketch proof of local existence. In section 5 we look at the model with indirect transfers only and prove the global existence and uniqueness of solutions. We also look at the asymptotic behavior of certain solutions. In section 6 we look at some numerical simulations and in section 7 we draw some conclusions. Finally in the appendix we give the numerical scheme used in section 6 .

\section{Mathematical models for direct and indirect transfers}

In this article we will use a model combining direct and indirect transfers. Let us start by considering the two of models separately.

\subsection{Purely direct cell-to-cell transfers}

We first assume (after a normalization) that the quantity of P-gp per cell, $r$, varies in the interval $I:=(0,1)$. Let $u(t, r)$ be the density of cells at time $t$ with respect to the quantity of P-gp $r$ on the surface membranes of cells. This means that

$$
\int_{r_{1}}^{r_{2}} u(t, r) d r
$$

is the number of cells with quantity of protein $r$ between $r_{1}$ and $r_{2}$ (for $r_{1}<r_{2}$ ), and the quantity of protein, which is attached to the cells is

$$
\int_{r_{-}}^{r_{+}} r u(t, r) d r
$$


The model describing the direct cell-to-cell transfers is the following

$$
\frac{\partial u(t, r)}{\partial t}=2 \widehat{\tau}[T(u(t, .))(r)-u(t, r)], \text { for } r \in I
$$

with initial data

$$
u(0, .)=u_{0} \in L_{+}^{1}(0,1) .
$$

The transfer operator $T: L_{+}^{1}(0,1) \rightarrow L_{+}^{1}(0,1)$ is defined by

$$
T(u)(r)= \begin{cases}\frac{1}{2} \frac{B(u, u)(r)}{\|u\|_{L^{1}}} & \text { if } u \neq 0 \\ 0 & \text { otherwise }\end{cases}
$$

where

$$
B(u, v)(r):=\int_{\mathbb{R}} \bar{u}(r+f(|\hat{r}|) \hat{r}) \bar{v}(r-(1-f(|\hat{r}|) \hat{r}) d \hat{r},
$$

Here

$$
\bar{u}(r):=\left\{\begin{array}{l}
u(r) \text { if } r \in(0,1) \\
0 \text { otherwise, }
\end{array}\right.
$$

and $f(\hat{r})$ is the transfer efficiency rate with $0 \leq f(\hat{r}) \leq 1$. $\widehat{\tau} \geq 0$ is the rate of transfer of protein per unit time. The above model was extensively studied in Hinow et al. [6]. In the part of this paper devoted to numerical simulations, we consider a generalized version of this transfer model. Namely we consider a model introduced in Magal et al. [11] with two efficiency fractions, and the bilinear operator $B$ replaced by

$$
\begin{aligned}
B(u, v)(r) & :=\int_{\mathbb{R}} \pi(\hat{r}) \bar{u}\left(r+f_{1}(|\hat{r}|) \hat{r}\right) \bar{v}\left(r-\left(1-f_{1}(|\hat{r}|) \hat{r}\right) d \hat{r}\right. \\
& +\int_{\mathbb{R}}(1-\pi(\hat{r})) \bar{u}\left(r+\left(1-f_{2}(|\hat{r}|)\right) \hat{r}\right) \bar{v}\left(r-f_{2}(|\hat{r}|) \hat{r}\right) d \hat{r} .
\end{aligned}
$$

Here $f_{1}(\hat{r})$ and $f_{2}(\hat{r})$ are the two transfer efficiency rates with $0 \leq f_{1}(\hat{r}) \leq$ $1 / 2$ and $0 \leq f_{2}(\hat{r}) \leq 1 / 2$. If two individuals whose difference in quantity is $\hat{r}$ are involved in a transfer, then the one with higher value loses $f_{1}(\hat{r})$ (respectively $f_{2}(\hat{r})$ ) times the difference of their $r$ values and the one with lower $r$ value gains exactly this amount with the probability $\pi$ (respectively with the probability $1-\pi)$, with

$$
\pi(\hat{r}):=\frac{\left[\frac{1}{2}-f_{2}(|\hat{r}|)\right]}{\left[1-\left(f_{1}+f_{2}\right)(|\hat{r}|)\right]} .
$$

\subsection{Purely indirect transfer}

Let $p(t)$ be the quantity of protein in the liquid surrounding the cells, at time $t$. We consider the following system describing the indirect transfers 


$$
\left\{\begin{aligned}
\partial_{t} u(t, r) & =\underbrace{\partial_{r}\left[\alpha^{-} \eta(r) u(t, r)\right]}_{\text {Protein released in the liquid }}-\underbrace{\partial_{r}\left[\alpha^{+} \eta(r) p(t) u(t, r)\right]}_{\text {Protein recaptured by cells }} \\
\frac{d p(t)}{d t} & =\underbrace{\alpha^{-} \int_{0}^{1} \eta(r) u(t, r) d r}-\underbrace{\alpha^{+} \int_{0}^{1} \eta(r) u(t, r) d r p(t)}_{\text {Protein released by the cells }} \\
u(0, .) & =u_{0}(.) \in L_{+}^{1}(0,1), \\
p(0) & =p_{0} \geq 0 .
\end{aligned}\right.
$$

where $\alpha^{+}>0$ is the rate at which P-gp is captured by the cells and $\alpha^{-}>0$ is the rate at which $\mathrm{P}$-gp is released in the liquid. The function $\eta(r)$ is the fraction (or the probability) of cells with a quantity of P-gp $r$ to capture or to release. Therefore it makes sense to assume that $\eta(0)=0$ since the cells with no protein can release no P-gp. Similarly, it is natural to assume that $\eta(1)=0$ since the cells with the maximum quantity of protein cannot capture any more P-gp.

In the rest of the paper we make the following assumption.

Assumption 2.1 We assume that $\eta \in C^{1}([0,1], \mathbb{R})$ with

$$
\eta(0)=\eta(1)=0
$$

and

$$
\eta(s)>0, \forall s \in(0,1) .
$$

Example 2.2 An example of such function $\eta(r)$ is

$$
\eta(s)=s^{n}(1-s)^{m}
$$

where $n \geq 1$ and $m \geq 1$ are two real numbers.

\section{Transport type equations and strongly contin- uous groups}

In this section we consider the semigroup properties of a linear size structured model. We refer to Webb [18] for more results and references on this topic. Let us focus on the following partial differential equation

$$
\left\{\begin{array}{l}
\partial_{t} u(t, s)+\partial_{s}(\eta(s) u(t, s))=f(t, s), \text { for } t \geq 0, \text { and } s \in I \\
u(0, .)=u_{0} \in L^{1}(I)
\end{array}\right.
$$

where the interval $I:=(0,1)$ and the map $t \rightarrow f(t,$.$) belongs to L^{1}\left((0, \tau), L^{1}(I)\right)$, for some $\tau>0$. Our aim will be to investigate the solutions of (3.1), by constructing an isometry $\Psi: L^{1}(\mathbb{R}) \rightarrow L^{1}(I)$ which relates it to the transport equation on $\mathbb{R}$. 
First we consider the differential equation associated with the characteristics. Since $\left|\eta^{\prime}(s)\right|$ is bounded, $\eta$ is Lipschitz continuous, and there exists a unique global solution of the ordinary differential equation

$$
\frac{\partial \Pi(t)\left(s_{0}\right)}{\partial t}=\eta\left(\Pi(t)\left(s_{0}\right)\right), \forall t \in \mathbb{R}, \text { and } \Pi(0)\left(s_{0}\right)=s_{0} \in \mathbb{R} .
$$

Therefore, we can define $\{\Pi(t)\}_{t \in \mathbb{R}}$ the flow generated by (3.2), namely $t \rightarrow$ $\Pi(t) s_{0}$ is the unique solution of (3.2) satisfying

$$
\Pi(0)\left(s_{0}\right)=s_{0} .
$$

Then the map $s \rightarrow \Pi(t)(s)$ is continuously differentiable and we set

$$
V(t, s):=\frac{\partial \Pi(t)(s)}{\partial s} .
$$

Then $V$ is solution of the differential equation

$$
\left\{\begin{array}{l}
\frac{\partial V(t, s)}{\partial t}=\eta^{\prime}(\Pi(t)(s)) V(t, s), \forall t \in \mathbb{R}, \\
V(0, s)=1 .
\end{array}\right.
$$

We have

$$
V(t, s)=\exp \left(\int_{0}^{t} \eta^{\prime}(\Pi(\tau)(s)) d \tau\right), \forall t \in \mathbb{R} .
$$

Consequently, for each $t \geq 0 s \mapsto V(t, s)$ is a positive function and

$$
\Pi\left(t, s_{0}\right) \leq \Pi\left(t, s_{1}\right) \text { for } s_{0} \leq s_{1} .
$$

Moreover, we have

$$
\frac{d}{d t}[\eta(\Pi(t)(s))-V(t, s) \eta(s)]=\eta^{\prime}(\Pi(t)(s))[\eta(\Pi(t)(s))-V(t, s) \eta(s)]
$$

thus

$$
\begin{aligned}
{[\eta(\Pi(t)(s))-V(t, s) \eta(s)] \quad } & =\exp \left(\int_{0}^{t} \eta^{\prime}(\Pi(l)(s)) d l\right)[\eta(\Pi(0)(s))-V(0, s) \eta(s)] \\
& =0
\end{aligned}
$$

which proves that

$$
\eta(\Pi(t)(s))=\eta(s) V(t, s) .
$$

Lemma 3.1 Let $s_{0} \in I$. Then

$$
t=\int_{s_{0}}^{\Pi(t)\left(s_{0}\right)} \frac{1}{\eta(r)} d r, \forall t \in \mathbb{R},
$$

and

$$
\lim _{s \rightarrow 1} \int_{s_{0}}^{s} \frac{1}{\eta(r)} d r=+\infty, \text { and } \lim _{s \rightarrow 0} \int_{s_{0}}^{s} \frac{1}{\eta(r)} d r=-\infty
$$


Proof. We have

$$
1=\frac{1}{\eta\left(\Pi(t)\left(s_{0}\right)\right)} \frac{d \Pi(t)\left(s_{0}\right)}{d t}, \forall t \in \mathbb{R},
$$

thus

$$
t=\int_{0}^{t} \frac{1}{\eta\left(\Pi(\sigma)\left(s_{0}\right)\right)} \frac{d \Pi(\sigma)\left(s_{0}\right)}{d \sigma} d \sigma=\int_{s_{0}}^{\Pi(t)\left(s_{0}\right)} \frac{1}{\eta(r)} d r .
$$

Since $\eta(s)>0, \forall s \in I, t \rightarrow+\infty$, we have

$$
\lim _{t \rightarrow+\infty} \Pi(t)\left(s_{0}\right)=1
$$

and

$$
\lim _{t \rightarrow-\infty} \Pi(t)\left(s_{0}\right)=0
$$

the result follows.

Let $s_{0} \in I$, and define the map $\Theta: I \rightarrow(-\infty,+\infty)$ by

$$
\Theta(s)=\int_{s_{0}}^{s} \frac{1}{\eta(l)} d l
$$

so using the previous lemma

$$
\Theta(I)=(-\infty,+\infty) .
$$

Lemma 3.2 For each $s \in I$ and each $t \in \mathbb{R}$ we have

$$
\Theta^{-1}(\Theta(s)-t)=\Pi(-t)(s) .
$$

Proof. Observe that

$$
\frac{\partial\left[\Theta^{-1}(\Theta(s)-t)\right]}{\partial t}=\frac{-1}{\Theta^{\prime}(\Theta(s)-t)}=-\eta\left(\Theta^{-1}(\Theta(s)-t)\right),
$$

and

$$
\left.\Theta^{-1}(\Theta(s)-t)\right|_{t=0}=s .
$$

therefore the equality (3.6) follows from the uniqueness of solutions of (3.2).

Define the map $\Psi: L^{1}(\mathbb{R}) \rightarrow L^{1}(I)$ as follows

$$
\Psi(\varphi)(s):=\varphi(\Theta(s)) \Theta^{\prime}(s)=\frac{\varphi(\Theta(s))}{\eta(s)} .
$$

It is bounded and invertible with $\Psi^{-1}: L^{1}(I) \rightarrow L^{1}(\mathbb{R})$ given by

$$
\Psi^{-1}(\varphi)(s)=\varphi\left(\Theta^{-1}(s)\right)\left(\Theta^{-1}\right)^{\prime}(s)=\frac{\varphi\left(\Theta^{-1}(s)\right)}{\Theta^{\prime}\left(\Theta^{-1}(s)\right)}=\varphi\left(\Theta^{-1}(s)\right) \eta\left(\Theta^{-1}(s)\right) .
$$


Moreover it is readily checked that

$$
\|\Psi(\varphi)\|_{L^{1}(I)}=\|\varphi\|_{L^{1}(\mathbb{R})}, \forall \varphi \in L^{1}(\mathbb{R}),
$$

therefore $\Psi$ and $\Psi^{-1}$ are isometries and

$$
\|\Psi\|_{\mathcal{L}\left(L^{1}(\mathbb{R})\right)}=\left\|\Psi^{-1}\right\|_{\mathcal{L}\left(L^{1}(\mathbb{R})\right)}=1 .
$$

Consider the linear operator $A: D(A) \subset L^{1}(I, \mathbb{R}) \rightarrow L^{1}(I, \mathbb{R})$ defined by

$$
A(\varphi)=-(\eta \varphi)^{\prime}
$$

with

$$
D(A)=\Psi\left(W^{1,1}(\mathbb{R})\right) .
$$

In order to make the domain of $A$ a little more explicit we have the following inclusion.

\section{Lemma 3.3}

$$
D(A) \subset\left\{\varphi \in L^{1}(I, \mathbb{R}):(\eta \varphi) \in W_{0}^{1,1}(I)\right\}
$$

where

$$
W_{0}^{1,1}(I)=\left\{\varphi \in W^{1,1}(I): \varphi(0)=\varphi(1)=0\right\} .
$$

Proof. Assume that $\varphi \in W^{1,1}(\mathbb{R})$, then for almost every $s \in(0,1)$

$$
\Psi(\varphi)(s)=\varphi(\Theta(s)) \Theta^{\prime}(s)=\frac{\varphi(\Theta(s))}{\eta(s)},
$$

thus

$$
(\eta \Psi(\varphi))^{\prime}(s)=(\varphi(\Theta(s)))^{\prime}=\varphi^{\prime}(\Theta(s)) \Theta^{\prime}(s)
$$

therefore

$$
\int_{I}\left|(\eta \Psi(\varphi))^{\prime}(s)\right| d s=\int_{I}\left|\varphi^{\prime}(\Theta(s))\right| \Theta^{\prime}(s) d s=\int_{\mathbb{R}}\left|\varphi^{\prime}(\sigma)\right| d \sigma .
$$

Moreover it is well known that

$$
\lim _{s \rightarrow \pm \infty} \varphi(s)=0
$$

therefore

$$
\lim _{s \rightarrow 0 \text { or } s \rightarrow 1} \eta(s) \Psi(\varphi)(s)=0
$$

The partial differential equation (3.1) can be regarded as the following abstract Cauchy problem.

$$
\left\{\begin{array}{l}
\frac{d u(t)}{d t}=A u(t)+f(t), t \geq 0 \\
u(0)=u_{0} \in L^{1}(I)
\end{array}\right.
$$


where $f \in L^{1}\left((0, \tau), L^{1}(I)\right)$.

We will show that $A$ generates a positive group of contractions in $L^{1}(I)$. We will see that group properties are inherited from the group properties of the transport equation on the real line. Let us consider the transport equation on the real line

$$
\left\{\begin{array}{l}
\partial_{t} v(t, s)+\partial_{s} v(t, s)=g(t, s), \text { for } t \geq 0 \text { and } s \in \mathbb{R} \\
v(0, .)=v_{0} \in L^{1}(\mathbb{R})
\end{array}\right.
$$

where the map $t \rightarrow g(t,$.$) belongs to L^{1}\left((0, \tau), L^{1}(\mathbb{R})\right)$. The solution of this problem is well understood. Consider the linear operator $\widehat{A}: D(\widehat{A}) \subset L^{1}(\mathbb{R}) \rightarrow$ $L^{1}(\mathbb{R})$ defined by

$$
\widehat{A} \varphi=-\varphi^{\prime}
$$

with

$$
D(\widehat{A})=W^{1,1}(\mathbb{R}) .
$$

One may observe that by construction

$$
D(A)=\Psi^{-1}(D(\widehat{A})) \Leftrightarrow D(\widehat{A})=\Psi(D(A)) .
$$

Then it is well known that $\widehat{A}$ is the infinitesimal generator of a strongly continuous group of contractions $\left\{T_{\widehat{A}}(t)\right\}_{t \in \mathbb{R}}$ on $L^{1}(\mathbb{R})$ which is defined by

$$
T_{\widehat{A}}(t)(\varphi)(s)=\varphi(s-t) .
$$

The partial differential equation (3.8) can be regarded as the following abstract Cauchy problem.

$$
\left\{\begin{array}{l}
\frac{d v(t)}{d t}=\widehat{A} v(t)+g(t), t \geq 0 \\
v(0)=v_{0} \in L^{1}(I)
\end{array}\right.
$$

where $g \in L^{1}\left((0, \tau), L^{1}(\mathbb{R})\right)$.

The unique mild solution of (3.9) is given by the following explicit formula

$$
\begin{aligned}
v(t, s) & =T_{\widehat{A}}(t) v_{0}+\int_{0}^{t} T_{\widehat{A}}(t-l) g(l) d l \\
& =\nu_{0}(s-t)+\int_{0}^{t} g(l, s-t+l) d l,
\end{aligned}
$$

for each $t \geq 0$ and almost every $s \in \mathbb{R}$.

Moreover $\sigma(\widehat{A})$ (the spectrum of $\widehat{A}$ ) is the set of purely imaginary complex numbers $\sigma(\widehat{A})=i \mathbb{R}$ and the resolvent set of $\widehat{A}$ is $\rho(\widehat{A}):=\mathbb{C} \backslash \sigma(\widehat{A})$. The resolvent operator of $\widehat{A}$ is defined for each $\lambda \in \rho(\widehat{A})$ by

$$
(\lambda I-\widehat{A})^{-1}(\varphi)=\left\{\begin{array}{l}
-\int_{0}^{+\infty} e^{-\lambda l} \varphi(s-l) d l, \text { if } \operatorname{Re}(\lambda)>0 \\
-\int_{0}^{+\infty} e^{\lambda l} \varphi(s+l) d l, \text { if } \operatorname{Re}(\lambda)<0 .
\end{array}\right.
$$

Furthermore we have the following relationship between $A$ and $\widehat{A}$. 
Lemma 3.4 We have following properties

(a) $\left.\Psi \widehat{A} \Psi^{-1}\right|_{D(A)}=A \Leftrightarrow \widehat{A}=\left.\Psi^{-1} A \Psi\right|_{D(\widehat{A})}$;

(b) $\sigma(A)=\sigma(\widehat{A}) \Leftrightarrow \rho(A)=\rho(\widehat{A})$;

(c) For each $\lambda \in \rho(A)$

$$
(\lambda I-A)^{-1}=\Psi(\lambda I-\widehat{A})^{-1} \Psi^{-1} \Leftrightarrow(\lambda I-A)=\Psi(\lambda I-\widehat{A}) \Psi^{-1} .
$$

Proof. Proof of (a). Let $\varphi \in D(A)$. Then for almost every $s \in \mathbb{R}$,

$$
\begin{aligned}
\widehat{A} \Psi^{-1}(\varphi)(s) & =-\left[\varphi\left(\Theta^{-1}\right) \eta\left(\Theta^{-1}\right)\right]^{\prime}(s) \\
& =-[\varphi \eta]^{\prime}\left(\Theta^{-1}(s)\right)\left[\Theta^{-1}\right]^{\prime}(s),
\end{aligned}
$$

and for $s \in I$

$$
\Psi \widehat{A} \Psi^{-1}(\varphi)(s)=-[\varphi \eta]^{\prime}\left(\Theta^{-1} \circ \Theta(s)\right)\left[\Theta^{-1}\right]^{\prime}(\Theta(s)) \Theta^{\prime}(s)=A(\varphi)(s) .
$$

Proof of (b) and (c). Assume that

$$
\lambda \varphi-A \varphi=\psi, \text { for } \varphi \in D(A)
$$

and since $A=\Psi \widehat{A} \Psi^{-1}$, the above equality is equivalent to

$$
\lambda \Psi^{-1} \varphi-\widehat{A} \Psi^{-1} \varphi=\Psi^{-1} \psi, \text { for } \varphi \in D(A)
$$

and since $\Psi^{-1}(D(A))=D(\widehat{A})$ and $\Psi^{-1} L^{1}(I)=L^{1}(\mathbb{R})$ the last equality is equivalent to

$$
\lambda \widehat{\varphi}-\widehat{A} \widehat{\varphi}=\widehat{\psi}, \text { for } \widehat{\varphi} \in D(\widehat{A})
$$

and $\widehat{\psi} \in L^{1}(\mathbb{R})$. The result follows.

As a consequence of the previous result we obtain the following proposition.

Proposition 3.5 The linear operator $A$ is the infinitesimal generator of a strongly continuous group $\left\{T_{A}(t)\right\}_{t \in \mathbb{R}}$ of bounded linear operators on $L^{1}(I)$. More precisely

$$
T_{A}(t)=\Psi T_{\widehat{A}}(t) \Psi^{-1}, \forall t \geq 0 .
$$

hence

$$
T_{A}(t)(\varphi)(s)=\varphi(\Pi(-t)(s)) V(-t, s) .
$$

and

$$
\left\|T_{A}(t)(\varphi)\right\|_{L^{1}(I)}=\|\varphi\|_{L^{1}(I)} .
$$


Remark 3.6 From the above formula we have

$$
\Psi^{-1} T_{A}(t)=T_{\widehat{A}}(t) \Psi^{-1}, \forall t \geq 0 .
$$

Let $u_{0} \in L^{1}(I)$ and $f \in L^{1}\left((0, \tau), L^{1}(I)\right)$. If we consider $u$ the mild solution of (3.7) which is given by

$$
u(t)=T_{A}(t) u_{0}+\int_{0}^{t} T_{A}(t-l) f(l) d l, \forall t \in[0, \tau],
$$

then $v(t)=\Psi^{-1} u(t)$ is the solution of (3.9) with

$$
v_{0}=\Psi^{-1} u_{0} \text { and } g=\Psi^{-1} f .
$$

Proof. The map $u \in C\left([0, \tau], L^{1}(I)\right)$ is the unique continuous function satisfying

$$
\int_{0}^{t} u(l) d s \in D(A), \forall t \in[0, \tau]
$$

and

$$
u(t)=u_{0}+A \int_{0}^{t} u(l) d l+\int_{0}^{t} f(l) d l, \forall t \in[0, \tau] .
$$

This last equality is equivalent to

$$
u(t)=u_{0}+\Psi \widehat{A} \Psi^{-1} \int_{0}^{t} u(l) d l+\int_{0}^{t} f(l) d l
$$

which is also equivalent to

$$
\Psi^{-1} u(t)=\Psi^{-1} u_{0}+\widehat{A} \int_{0}^{t} \Psi^{-1} v(l) d l+\int_{0}^{t} \Psi^{-1} f(l) d l .
$$

The result follows from the uniqueness of the weak solution.

From the previous results, it follows that $u(t)$ is the unique mild solution of (3.1) if and only if $v(t)=\Psi^{-1} u(t)$ is the unique mild solution of (3.8) with $v_{0}=\Psi^{-1} u_{0}$ and $g(t, s)=\Psi^{-1}(f(t,)).(s)$. So from (3.10) we have

$$
\begin{aligned}
v(t, s) & =\Psi^{-1}\left(u_{0}\right)(s-t)+\int_{0}^{t} \Psi^{-1}(f(l, .))(s-t+l) d l \\
& =u_{0}\left(\Theta^{-1}(s-t)\right)\left(\Theta^{-1}\right)^{\prime}(s-t)+\int_{0}^{t} f\left(l, \Theta^{-1}(s-t+l)\right)\left(\Theta^{-1}\right)^{\prime}(s-t+l) d l \\
& =u_{0}\left(\Theta^{-1}(s-t)\right) \eta\left(\Theta^{-1}(s-t)\right)+\int_{0}^{t} f\left(l, \Theta^{-1}(s-t+l)\right) \eta\left(\Theta^{-1}(s-t+l)\right) d l
\end{aligned}
$$

and

$$
u(t, s)=\Psi(v(t, s))(s)=v(t, \Theta(s)) \Theta^{\prime}(s)=\frac{v(t, \Theta(s))}{\eta(s)}
$$


$\mathrm{SO}$

$$
\begin{aligned}
u(t, s)= & u_{0}\left(\Theta^{-1}(\Theta(s)-t)\right) \frac{\eta\left(\Theta^{-1}(\Theta(s)-t)\right)}{\eta(s)}+ \\
& \int_{0}^{t} f\left(l, \Theta^{-1}(\Theta(s)-t+l)\right) \frac{\eta\left(\Theta^{-1}(\Theta(s)-t+l)\right)}{\eta(s)} d l
\end{aligned}
$$

thus by using (3.6) we obtain

$$
u(t, s)=u_{0}(\Pi(-t)(s)) \frac{\eta(\Pi(-t)(s))}{\eta(s)}+\int_{0}^{t} f(l, \Pi(l-t)(s)) \frac{\eta(\Pi(l-t)(s))}{\eta(s)} d l
$$

now by using (3.3) we obtain

Proposition 3.7 The unique weak solution of (3.1) is given by

$$
u(t, s)=u_{0}(\Pi(-t)(s)) V(-t, s)+\int_{0}^{t} f(l, \Pi(l-t)(s)) V(l-t, s) d l
$$

Example 3.8 Assume that

$$
\eta(s)=s(1-s)
$$

Fix $s_{0}=1 / 2$. We have

$$
\Theta(s)=\int_{1 / 2}^{s} \frac{1}{l(1-l)} d l=\int_{1 / 2}^{s} \frac{1}{l}+\frac{1}{1-l} d l=[\ln (l)-\ln (1-l)]_{1 / 2}^{s}
$$

thus

$$
\Theta(s)=\ln \left(\frac{s}{1-s}\right)
$$

Moreover

$$
\begin{aligned}
\Theta(s) & =y \Leftrightarrow \ln \left(\frac{s}{1-s}\right)=y \Leftrightarrow s=\exp (y)(1-s) \\
& \Leftrightarrow s=\frac{\exp (y)}{1+\exp (y)}
\end{aligned}
$$

and

$$
\eta\left(\Theta^{-1}(s)\right)=\frac{\exp (y)}{1+\exp (y)}\left[1-\frac{\exp (y)}{1+\exp (y)}\right]
$$

\section{Weak and mild solutions}

The full model combining direct and indirect transfers can be rewritten as the following abstract Cauchy problem

$$
\left\{\begin{array}{l}
\frac{d u(t)}{d t}=\left(\alpha^{+} p(t)-\alpha^{-}\right) A u(t)+2 \widehat{\tau}[T(u(t))-u(t)] \\
\frac{d p(t)}{d t}=-\left(\alpha^{+} p(t)-\alpha^{-}\right) \int_{I} \eta(r) u(t, r) d r
\end{array}\right.
$$


with initial data

$$
u(0)=u_{0} \in L_{+}^{1}(I) \text { and } p(0)=p_{0} \geq 0 .
$$

The goal of this section is to define a notion of mild and weak solution for the first equation in (4.1). The last part of the section will be devoted to providing a nonlinear variation of constants formula for the first equation of system (4.1).

Given a function $q \in C((0, \tau), \mathbb{R})$ (we will fix later on $\left.q:=\alpha^{+} p-\alpha^{-}\right)$we consider the abstract non-homogeneous Cauchy problem

$$
\frac{d u(t)}{d t}=q(t) A u(t)+f(t), \text { for } t \in[0, \tau], \text { with } u(0)=u_{0} \in L^{1}(I),
$$

where $f \in L^{1}\left((0, \tau), L^{1}(I)\right)$ (for some $\left.\tau>0\right)$.

Throughout this article, we systematically use the following notation

$$
Q(t):=\int_{0}^{t} q(s) d s
$$

whenever

$$
q \in C([0, \tau], \mathbb{R}) .
$$

We start with the notion of mild solution.

Definition 4.1 We will say that $u \in C\left([0, \tau], L^{1}(I)\right)$ is a mild solution of (2.1) if

$$
u(t)=T_{A}(Q(t)) u_{0}+\int_{0}^{t} T_{A}(Q(t)-Q(s)) f(s) d s, \forall t \in[0, \tau] .
$$

Let us prove that such a mild solution is nothing but a weak solution. We first observe that

$$
\int_{0}^{t} q(s) T_{A}(Q(s)) u_{0} d s=\int_{0}^{Q(t)} T_{A}(s) u_{0} d s .
$$

So using the fact that (see Pazy [14])

$$
\int_{0}^{t} T_{A}(s) u_{0} d s \in D(A)
$$

and the classical formula

$$
T_{A}(t) u_{0}=u_{0}+A \int_{0}^{t} T_{A}(s) u_{0} d s
$$

we deduce that

$$
\int_{0}^{t} q(s) T_{A}(Q(s)) u_{0} d s \in D(A)
$$


and

$$
A \int_{0}^{t} q(s) T_{A}(Q(s)) u_{0} d s=T_{A}(Q(t)) u_{0}-u_{0}
$$

Furthermore, by using Fubini's theorem

$$
\int_{0}^{t} q(s) \int_{0}^{s} T_{A}(Q(s)-Q(l)) f(l) d l d s=\int_{0}^{t} \int_{l}^{t} q(s) T_{A}(Q(s)-Q(l)) f(l) d s d l
$$

so by setting $\sigma=Q(s)-Q(l)$, we obtain

$$
\int_{0}^{t} q(s) \int_{0}^{s} T_{A}(Q(s)-Q(l)) f(l) d l d s=\int_{0}^{t} \int_{0}^{Q(t)-Q(l)} T_{A}(\sigma) f(l) d \sigma d l
$$

and since $A$ a closed linear operator, we have

$$
\int_{0}^{t} q(s) \int_{0}^{s} T_{A}(Q(s)-Q(l)) f(l) d l d s \in D(A)
$$

and

$A \int_{0}^{t} q(s) \int_{0}^{s} T_{A}(Q(s)-Q(l)) f(l) d l d s=\int_{0}^{t} T_{A}(Q(t)-Q(s)) f(s) d s-\int_{0}^{t} f(s) d s$.

Therefore each mild solution of (4.2) is a weak solution of (4.2) in the following sense.

Definition 4.2 We will say that $u \in C\left([0, \tau], L^{1}(I)\right)$ is a weak solution of (4.2) if

$$
\int_{0}^{t} q(s) u(s) d s \in D(A), \forall t \in[0, \tau]
$$

and

$$
u(t)=u_{0}+A \int_{0}^{t} q(s) u(s) d s+\int_{0}^{t} f(s) d s, \forall t \in[0, \tau] .
$$

It is natural to ask about uniqueness of such weak solutions.

Proposition 4.3 (Uniqueness) There exists at most one weak solution of (4.2).

Proof. Let $u_{0} \in L^{1}(I)$ and $f \in L^{1}\left((0, \tau), L^{1}(I)\right)$ (for some $\tau>0$ ). Assume that there exist $u_{1}$ and $u_{2}$ two weak solution of $(4.2)$ on $[0, \tau]$. Then $u=u_{1}-u_{2}$ is a weak solution with $u_{0}=0$ and $f=0$.

Without loss of generality we can assume that $q \geq 0$. Assume that first $t \rightarrow Q(t)$ is strictly increasing function. Set

$$
v(t):=u\left(Q^{-1}(t)\right), \forall t \in[0, Q(\tau)] .
$$

Then

$$
\int_{0}^{t} q(s) u(s) d s=\int_{0}^{t} q(s) v(Q(s)) d s=\int_{0}^{Q(t)} v(l) d l
$$


and

$$
v(Q(t))=u(t)=A \int_{0}^{Q(t)} v(l) d l
$$

so

$$
v(t)=A \int_{0}^{t} v(l) d l, \forall t \in[0, Q(\tau)]
$$

Now since $A$ is the infinitesimal generator of strongly continuous semigroup, it follows that $v=0$, so $u=0$. When $t \rightarrow Q(t)$ is only non-decreasing function, we use the same arguments, and the fact that we can find $v \in C\left([0, Q(\tau)], L^{1}(I)\right)$ such that

$$
v(Q(t))=u(t), \forall t \in[0, \tau] .
$$

The connection between the classical non-homogeneous Cauchy problem with $q=1$ and the Cauchy problem $q \neq 1$ is described in the following lemma.

\section{Lemma 4.4 (Change of variable)}

Let $u_{0} \in L^{1}(I)$ and $f \in L^{1}\left((0, \tau), L^{1}(I)\right)$. Then

$$
v(t)=T_{A}(t) u_{0}+\int_{0}^{t} T_{A}(t-s) f(s) d s, \forall t \in[0, Q(\tau)]
$$

if and only if

$$
v(Q(t))=T_{A}(Q(t)) u_{0}+\int_{0}^{t} T_{A}(Q(t)-Q(l)) q(l) f(Q(l)) d l, \forall t \in[0, \tau] .
$$

Proof. If $v$ satisfies (4.6) then

$$
v(Q(t))=u_{0}+A \int_{0}^{Q(t)} v(l) d l+\int_{0}^{Q(t)} g(l) d l, \forall t \in[0, \tau] .
$$

By setting $l=Q(s)$, we obtain

$$
v(Q(t))=u_{0}+A \int_{0}^{t} q(s) v(Q(s)) d s+\int_{0}^{t} q(s) g(Q(s)) d s, \forall t \in[0, \tau],
$$

and by uniqueness of the weak solutions, the result follows.

Proposition 4.5 Let $u_{0} \in L^{1}(I), f \in L^{1}\left((0, \tau), L^{1}(I)\right)$ and $\lambda \in \mathbb{R}$. Then

$$
u(t)=e^{\lambda t} T_{A}(Q(t)) u_{0}+\int_{0}^{t} e^{\lambda(t-s)} T_{A}(Q(t)-Q(s)) f(s) d s, \forall t \in[0, \tau],
$$

is the unique weak solution of

$$
\frac{d u(t)}{d t}=q(t) A u(t)+\lambda u(t)+f(t) \text { for } t \in[0, \tau] \text { with } u(0)=u_{0} \in L^{1}(I) .
$$


Proof. Set

$$
S_{A}(t) u:=\int_{0}^{t} T_{A}(s) u d s, \forall t \geq 0, \forall u \in L^{1}(I) .
$$

For $t, s \in[0, \tau]$ with $t \geq s$, and for $u \in L^{1}(I)$ we obtain by integrating by parts

$$
\begin{aligned}
\int_{s}^{t} q(l) e^{\lambda l} T_{A}(Q(l)-Q(s)) u d l & =\left[e^{\lambda l} S_{A}(Q(l)-Q(s)) u\right]_{s}^{t}-\lambda \int_{s}^{t} e^{\lambda l} S_{A}(Q(l)-Q(s)) u d l \\
& =e^{\lambda t} S_{A}(Q(t)-Q(s)) u-\lambda \int_{s}^{t} e^{\lambda l} S_{A}(Q(l)-Q(s)) u d l .
\end{aligned}
$$

So

$$
\int_{s}^{t} q(l) e^{\lambda l} T_{A}(Q(l)-Q(s)) u d l \in D(A)
$$

and

$$
\begin{aligned}
A \int_{s}^{t} q(l) e^{\lambda l} T_{A}(Q(l)-Q(s)) u d l & =e^{\lambda t} A S_{A}(Q(t)-Q(s)) u-\lambda \int_{s}^{t} e^{\lambda l} A S_{A}(Q(l)-Q(s)) u d l \\
& =e^{\lambda t}\left[T_{A}(Q(t)-Q(s)) u-u\right]-\lambda \int_{s}^{t} e^{\lambda l}\left[T_{A}(Q(l)-Q(s)) u-u\right] d l
\end{aligned}
$$

Therefore

$$
\begin{aligned}
A \int_{s}^{t} q(l) e^{\lambda(l-s)} T_{A}(Q(l)-Q(s)) u d l & =e^{\lambda(t-s)} T_{A}(Q(t)-Q(s)) u-u \\
& -\int_{s}^{t} \lambda e^{\lambda(l-s)} T_{A}(Q(l)-Q(s)) u d l .
\end{aligned}
$$

By using Fubini's theorem for each $t \in[0, \tau]$,

$$
\int_{0}^{t} q(s) \int_{0}^{s} e^{\lambda(s-l)} T_{A}(Q(s)-Q(l)) f(l) d l d s=\int_{0}^{t} \int_{l}^{t} q(s) e^{\lambda(s-l)} T_{A}(Q(s)-Q(l)) f(l) d s d l,
$$

and since $A$ is closed, (4.9) implies that

$$
\int_{0}^{t} q(s) \int_{0}^{s} e^{\lambda(s-l)} T_{A}(Q(s)-Q(l)) f(l) d l d s \in D(A) .
$$

Moreover by using (4.10)

$$
\begin{aligned}
A \int_{0}^{t} q(s) \int_{0}^{s} e^{\lambda(s-l)} T_{A}(Q(s)-Q(l)) f(l) d l d s= & \int_{0}^{t} e^{\lambda(t-l)} T_{A}(Q(t)-Q(l)) f(l) d l-\int_{0}^{t} f(l) d l \\
& -\int_{0}^{t} \int_{l}^{t} \lambda e^{\lambda(\sigma-l)} T_{A}(Q(\sigma)-Q(l)) f(l) d \sigma d l .
\end{aligned}
$$

By using again Fubini's theorem for the last integral of the above equation, we obtain

$$
\begin{aligned}
A \int_{0}^{t} q(s) \int_{0}^{s} e^{\lambda(s-l)} T_{A}(Q(s)-Q(l)) f(l) d l d s= & \int_{0}^{t} e^{\lambda(t-l)} T_{A}(Q(t)-Q(l)) f(l) d l-\int_{0}^{t} f(l) d l \\
& -\int_{0}^{t} \int_{0}^{s} \lambda e^{\lambda(s-l)} T_{A}(Q(s)-Q(l)) f(l) d l d s .
\end{aligned}
$$


It follows that if $u(t)$ defined by (4.7) then $u$ is a weak solution of (4.8).

Sketch of proof for the local existence: Let us now reconsider (4.1). By using Proposition 4.5 with $\lambda=-2 \widehat{\tau}$ the mild solution of (4.1) is given by

$$
\left\{\begin{array}{l}
u(t)=e^{-2 \widehat{\tau} t} T_{A}(Q(t)) u_{0}+\int_{0}^{t} e^{-2 \widehat{\tau}(t-s)} T_{A}(Q(t)-Q(s)) 2 \widehat{\tau} T(u(s)) d s \\
q(t)=\exp \left(-\alpha^{+} \int_{0}^{t} \int_{I} \eta(r) u(l, r) d r d l\right) q_{0}
\end{array}\right.
$$

Remark 4.6 It not possible to use a fixed point technique directly on the above system, since $t \rightarrow T_{A}(t) u$ is $C^{1}$ if and only if $u$ belongs to the domain of $A$, and we cannot control the range of $T$ (since $T$ contains non local terms).

Assume for example that $q_{0}>0$. Then by using the second equation of (4.11) we deduce that

$$
q(t)>0, \forall t \geq 0
$$

The map $t \rightarrow Q(t)=\int_{0}^{t} q(s) d s$ is monotone and (possibly bounded) therefore we define

$$
Q^{+}:=\sup _{t \geq 0} Q(t)
$$

and we define $Q^{-1}:\left[0, Q^{+}\right) \rightarrow \mathbb{R}_{+}$the inverse function of $Q(t)$.

By using the fact that $T$ is homogeneous we deduce that

$$
w(t)=e^{2 \widehat{\tau} t} u(t) \Leftrightarrow u(t)=e^{-2 \widehat{\tau} t} w(t)
$$

and system (4.11) becomes

$$
\left\{\begin{array}{l}
w(t)=T_{A}(Q(t)) u_{0}+\int_{0}^{t} T_{A}(Q(t)-Q(s)) 2 \widehat{\tau} T(w(s)) d s \\
q(t)=\exp \left(-\alpha^{+} \int_{0}^{t} e^{-2 \widehat{\tau} l} \int_{I} \eta(r) w(l, r) d r d l\right) q_{0} .
\end{array}\right.
$$

Setting

$$
v(t)=w\left(Q^{-1}(t)\right)\left(\text { for } t \in\left[0, Q^{+}\right)\right) \Leftrightarrow v(Q(t))=w(t)\left(\text { for } t \in \mathbb{R}_{+}\right)
$$

then by using the change of variable in Lemma 4.4, the first equation of system (4.12) becomes

$$
v(t)=T_{A}(t) v_{0}+\int_{0}^{t} T_{A}(t-s) \frac{2 \widehat{\tau} T(v(s))}{q\left(Q^{-1}(s)\right)} d s .
$$

Set

$$
\widehat{q}(t):=q\left(Q^{-1}(t)\right)
$$

We observe that

$$
\int_{0}^{t} \frac{1}{\widehat{q}(s)} d s=\int_{0}^{t} \frac{1}{q\left(Q^{-1}(s)\right)} d s=\int_{0}^{t} Q^{-1}(s)^{\prime} d s=Q^{-1}(t)
$$


and

$$
\begin{aligned}
\widehat{q}(t) & =\exp \left(-\alpha^{+} \int_{0}^{Q^{-1}(t)} e^{-2 \widehat{\tau} l} \int_{I} \eta(r) w(l, r) d r d l\right) q_{0} \\
& =\exp \left(-\alpha^{+} \int_{0}^{Q^{-1}(t)} e^{-2 \widehat{\tau} l} \int_{I} \eta(r) v(Q(l), r) d r d l\right) q_{0}
\end{aligned}
$$

setting $\sigma=Q(l)$ we obtain

$$
\widehat{q}(t)=\exp \left(-\alpha^{+} \int_{0}^{t} e^{-2 \widehat{\tau} Q^{-1}(\sigma)} \int_{I} \eta(r) v(\sigma, r) d r d \sigma\right) q_{0} .
$$

So finally by using (4.10) we obtain the system

$$
\left\{\begin{array}{l}
v(t)=T_{A}(t) u_{0}+\int_{0}^{t} T_{A}(t-s) \frac{2 \widehat{\tau} T(v(s))}{\widehat{q}(s)} d s . \\
\widehat{q}(t)=\exp \left(-\alpha^{+} \int_{0}^{t} e^{-2 \widehat{\tau} \int_{0}^{\sigma} \frac{1}{\widehat{q}(s)} d s} \int_{I} \eta(r) v(\sigma, r) d r d \sigma\right) q_{0} .
\end{array}\right.
$$

and we look for the solutions of the above system only for $t \in\left[0, Q^{+}\right)$.

We observe that the fixed point problem is singular when $\widehat{q}(t)$ converges to 0 . Nevertheless, one may use the above fixed point problem to obtain the local existence of solutions. This problem as well as the semiflow problem will be studied elsewhere.

\section{$5 \quad$ Indirect transfer model}

In this section we consider the case $\widehat{\tau}=0$ which corresponds to the following abstract Cauchy problem

$$
\left\{\begin{array}{l}
\frac{d u(t)}{d t}=\left(\alpha^{+} p(t)-\alpha^{-}\right) A u(t) \\
\frac{d p(t)}{d t}=-\left(\alpha^{+} p(t)-\alpha^{-}\right) \int_{I} \eta(r) u(t, r) d r
\end{array}\right.
$$

and by using again $q(t):=\left(\alpha^{+} p(t)-\alpha^{-}\right)$the mild solution is given by

$$
\left\{\begin{array}{l}
u(t)=T_{A}(Q(t)) u_{0} \\
q(t)=\exp \left(-\alpha^{+} \int_{0}^{t} \int_{I} \eta(r) u(l, r) d r d l\right) q_{0} .
\end{array}\right.
$$

\subsection{Existence and uniqueness of solutions}

In order to deal with the existence of solutions, we can rewrite the second equation of (5.2) as the following ordinary differential equation

$$
Q^{\prime}(t)=\exp \left(-\alpha^{+} \int_{0}^{t} \int_{I} \eta(r) T_{A}(Q(l))\left(u_{0}\right)(r) d r d l\right) q_{0} .
$$

with

$$
Q(0)=0 .
$$

In order to obtain a global existence result we have the following properties 
Lemma 5.1 We have the following estimates

(a) The map $t \rightarrow \int_{I} T_{A}(t)\left(u_{0}\right)(r) d r$ is $C^{1}$ and

$$
\frac{d \int_{I} T_{A}(t)\left(u_{0}\right)(r) d r}{d t}=0 .
$$

(b) The map $t \rightarrow \int_{I} \eta(r) T_{A}(t)\left(u_{0}\right)(r) d r$ is $C^{1}$ and

$$
\frac{d \int_{I} \eta(r) T_{A}(t)\left(u_{0}\right)(r) d r}{d t}=\int_{I} \eta(r) \eta^{\prime}(r) T_{A}(t)\left(u_{0}\right)(r) d r
$$

(c) The map $t \rightarrow \int_{I} r T_{A}(t)\left(u_{0}\right)(r) d r$ is $C^{1}$ and

$$
\frac{d \int_{I} r T_{A}(t)\left(u_{0}\right)(r) d r}{d t}=q(t) \int_{I} \eta(r) T_{A}(t)\left(u_{0}\right)(r) d r .
$$

Proof. Let $\chi \in C^{1}(I)$. Assume first that $u_{0} \in D(A)$. Let $u(t, r)=T_{A}(t)\left(u_{0}\right)(r)$ then

$$
\begin{aligned}
\frac{d}{d t} \int_{I} \chi(r) u(t, r) d r & =-\int_{I} \chi(r) \frac{\partial}{\partial r}(\eta(r) u(t, r)) d r \\
& =-[\chi(r) \eta(r) u(t, r)]_{0}^{1}+\int_{I} \chi^{\prime}(r) \eta(r) u(t, r) d r
\end{aligned}
$$

and since $\eta(0)=\eta(1)=0$

$$
\frac{d}{d t} \int_{I} \chi(r) u(t, r) d r=\int_{I} \chi^{\prime}(r) \eta(r) u(t, r) d r
$$

the result follows by density of $D(A)$ in $L^{1}(I)$.

Theorem 5.2 Let Assumption 2.1 be satisfied. Then the system (5.1) generates a unique continuous semiflow $\{U(t)\}_{t \geq 0}$ on $L_{+}^{1}(I, \mathbb{R}) \times[0,+\infty)$. That is to say that for each $\left(u_{0}, p_{0}\right) \in L_{+}^{1}(I, \mathbb{R}) \times[0,+\infty)$, there exists a unique mild (or weak) solution $(u(),. p().) \in C\left([0,+\infty), L_{+}^{1}(I, \mathbb{R}) \times[0,+\infty)\right)$. Moreover if we define

$$
U(t)\left(u_{0}, p_{0}\right):=(u(t), p(t)), \forall t \geq 0,
$$

then $U$ is a continuous semiflow. That is to say that

(i) $U(0)=I$ and $U(t) U(s)=U(t+s), \forall t, s \geq 0$;

(ii) The map $\left(t,\left(u_{0}, p_{0}\right)\right) \rightarrow U(t)\left(u_{0}, p_{0}\right)$ is a continuous map from $[0,+\infty) \times$ $L_{+}^{1}(I, \mathbb{R}) \times[0,+\infty)$ into $L_{+}^{1}(I, \mathbb{R}) \times[0,+\infty)$.

Proof. It is sufficient to prove the global existence of solutions of the integral equation

$$
Q^{\prime}(t)=\exp \left(-\alpha^{+} \int_{0}^{t} F(Q(l)) d l\right) q_{0} .
$$


with

$$
Q(0)=0
$$

with

$$
F(t)=\int_{I} \eta(r) T_{A}(t)\left(u_{0}\right)(r) d r
$$

and from Lemma 5.1 we deduce that $F$ is Lipschitz continuous. The result follows.

Conservation properties: By using again Lemma 5.1 it follows that the total number of cells is conserved

$$
\int_{I} u(t, r) d r=\int_{I} u_{0}(r) d r, \forall t \geq 0,
$$

and the total quantity of protein is conserved

$$
\int_{I} r u(t, r) d r+p(t)=\int_{I} r u_{0}(r) d r+p_{0}, \forall t \geq 0 .
$$

Equilibria: Each distribution $\bar{u} \in L_{+}^{1}(I)$ is an equilibrum solution with

$$
\bar{p}=\frac{\alpha^{-}}{\alpha^{+}} .
$$

\subsection{Asymptotic behavior}

To study the asymptotic behavior, we first set

$$
w(t)=\int_{I} \eta(r) u(t, r) d r \text { and } w_{0}=\int_{I} \eta(r) u_{0}(r) d r
$$

then

$$
q(t)=e^{-\alpha^{+} \int_{0}^{t} w(l) d l} q_{0}
$$

Now by using the fact that $u(t, r)$ is a weak solution we deduce that

$$
w(t)=w_{0}(r)+\int_{I} \eta(r) A \int_{0}^{t} q(s) u(s, r) d s d r .
$$

By integrating by parts and by using Fubini's theorem we obtain

$$
\begin{aligned}
\int_{I} \eta(r) A \int_{0}^{t} q(s) u(s, r) d s d r & =\int_{0}^{t} q(s) \int_{I} \eta(r)^{\prime} \eta(r) u(s, r) d r d s \\
& =\int_{0}^{t} q(s) \int_{I} \eta(r)^{\prime} \eta(r) T_{A}(Q(t))\left(u_{0}\right)(r) d r d s
\end{aligned}
$$

Set

$$
H(t):=\int_{I} \eta(r)^{\prime} \eta(r) T_{A}(t)\left(u_{0}\right)(r) d r d s .
$$

Therefore we obtain a system

$$
\left\{\begin{array}{l}
q(t)=e^{-\alpha^{+} \int_{0}^{t} w(l) d l} q_{0} \\
w(t)=w_{0}+\int_{0}^{\int_{0}^{t} q(s) d s} H(\sigma) d \sigma .
\end{array}\right.
$$


The following theorem is basically saying that some solutions converge to an equilibrium that depends on the initial distribution. This result will be observed numerically in Figure 3 for (a) and in Figure 4 for (b).

Theorem 5.3 Let Assumption 2.1 be satisfied. Then the following properties hold

(a) If

$$
q_{0}=\left(\alpha^{+} p_{0}-\alpha^{-}\right)>0,
$$

and

$$
w_{0}+\int_{0}^{+\infty} H(s) d s<0
$$

then the map $q(t)=\left(\alpha^{+} p(t)-\alpha^{-}\right)$is a strictly positive function which is integrable. Moreover

$$
\begin{aligned}
Q(\infty) & :=\int_{0}^{+\infty} q(s) d s \in(0,+\infty), \\
\lim _{t \rightarrow \infty} p(t) & =\frac{\alpha^{-}}{\alpha^{+}} \quad \text { and } \\
\lim _{t \rightarrow+\infty} u(t) & =T_{A}(Q(\infty)) u_{0} .
\end{aligned}
$$

(b) If

$$
q_{0}=\left(\alpha^{+} p_{0}-\alpha^{-}\right)<0,
$$

and

$$
w_{0}-\int_{-\infty}^{0} H(s) d s<0
$$

then the map $q(t)=\left(\alpha^{+} p(t)-\alpha^{-}\right)$is a strictly negative function which is integrable. Moreover

$$
Q(\infty):=\int_{0}^{+\infty} q(s) d s \in(-\infty, 0)
$$

and again (5.7) and (5.8) hold.

Proof. Let $u_{0} \geq 0$. Let us prove (a) (the case (b) is similar). Suppose that $q_{0}>0$. Then $t \rightarrow q(t)$ is non-increasing and remains positive. Set

$$
q_{\infty}:=\lim _{t \rightarrow+\infty} q(t) \geq 0
$$

The function $t \rightarrow \int_{0}^{t} q(s) d s$ is increasing. Assume by contradiction that

$$
Q(\infty)=+\infty .
$$

then by using the $w$-equation of system (5.5) we obtain

$$
\liminf _{t \rightarrow+\infty} w(t) \leq w_{0}+\int_{0}^{+\infty} H(s) d s
$$


and condition (5.6) implies that

$$
\liminf _{t \rightarrow+\infty} w(t)<0
$$

which is impossible since $w(t) \geq 0, \forall t \geq 0$. This implies that

$$
Q(\infty)<+\infty
$$

so we must have in particular $q_{\infty}=0$.

\section{Numerical simulations}

In this section we assume the transfer efficiency rates $f_{1}$ and $f_{2}$ are defined as

$$
f_{i}\left(r_{j}-r_{k}\right)=\left\{\begin{array}{l}
\bar{f}_{i} \text { if }\left(r_{j}-r_{k}\right) \in\left(\delta_{\min }, \delta_{\max }\right) \\
\epsilon \bar{f}_{i} \quad \text { otherwise }
\end{array}\right.
$$

where $\bar{f}_{i}$, for $\mathrm{i}=1$ and $\mathrm{i}=2$, represent the constant fraction of transferred quantity.

Remark 6.1 $\epsilon=0$ means that the transfer occurs only if $\delta_{\min }<r_{j}-r_{k}<\delta_{\max }$. $\epsilon \in(0,1)$ means that a low quantity is transferred when the difference $r_{j}-r_{k}$ is either very low or very large.

Moreover the numerical simulations are obtained by using the numerical scheme (8.3) and (8.4). We present four experiments to show the effect of the direct and indirect transfers.

In the last experiment (Experiment 4), we calibrate and validate the model using experimental data developed in the work of ([13]). At each time step, we use an uniform grid over the interval $[0,1]$.

\begin{tabular}{llll}
\hline Description & Experiment 1 & Experiment 2 & Experiment 3 \\
\hline \hline Transfer & Indirect & Indirect & Direct and indirect \\
Cells & Sensitive & Resistant & Sensitive and resistant \\
liquid & $\mathrm{p}(0)=0.6$ & $\mathrm{p}(0)=0.4$ & $\mathrm{p}(0)=0.6$ \\
$u_{0}(r)$ & $(6.2)$ & $(6.3)$ & $(6.6)$ \\
$\alpha^{+}$ & 0.75 & 0.75 & 0.75 \\
$\alpha^{-}$ & $0.375=\alpha^{+} / 2$ & 0.375 & 0.375 \\
$\eta(r)=r(1-r)$ & & & \\
$\widehat{\tau}$ & 0 & 0 & 0.95 \\
$\bar{f}_{1}$ & 0.48 & 0.48 & 0.48 \\
$\bar{f}_{2}$ & 0.07 & 0.07 & 0.07 \\
$\epsilon \bar{f}_{1}$ & 0.05 & 0.05 & 0.05 \\
$\epsilon \bar{f}_{2}$ & 0.007 & 0.007 & 0.007 \\
\hline
\end{tabular}

Table 1: List of parameters used in each experiment. 


\subsection{Experiment 1}

In this experiment we consider a numerical simulation corresponding to sensitive cells in a dish. Those cells have a low level of P-gp expression. In this illustration the quantity of $\mathrm{P}-\mathrm{gp} r$ for sensitive cells will vary in between 0 and 0.25 at time $t=0$. We assume that the surrounding liquid contains a quantity of protein given by $p(0)=0.6$. We use the indirect transfer model only (i.e. with $\widehat{\tau}=0$ ) with $q(0)>0$. This experiment was run with initial data $u_{0}$ chosen as follows

$$
u(0, r)=\left\{\begin{array}{lll}
1 & \text { if } & 0 \leq r \leq 0.25 \\
0 & \text { if } & 0.25 \leq r \leq 1
\end{array}\right.
$$

Since $q_{0}=\left(\alpha^{+} p_{0}-\alpha^{-}\right)>0$, the map $q(t)=\left(\alpha^{+} p(t)-\alpha^{-}\right)$is a strictly positive function which is integrable. We observe that the cells take up protein from the surrounding environmental liquid to increase their total quantity of protein. In figure (3) we can see that the successive curves converge to a steady state. Moreover as time $t$ goes to infinity we have

$$
\begin{aligned}
Q(\infty) & :=\int_{0}^{+\infty} q(s) d s \in(0,+\infty), \\
\lim _{t \rightarrow \infty} p(t) & =\frac{\alpha^{-}}{\alpha^{+}} \text {and } \\
\lim _{t \rightarrow+\infty} u(t) & =T_{A}(Q(\infty)) u_{0} .
\end{aligned}
$$




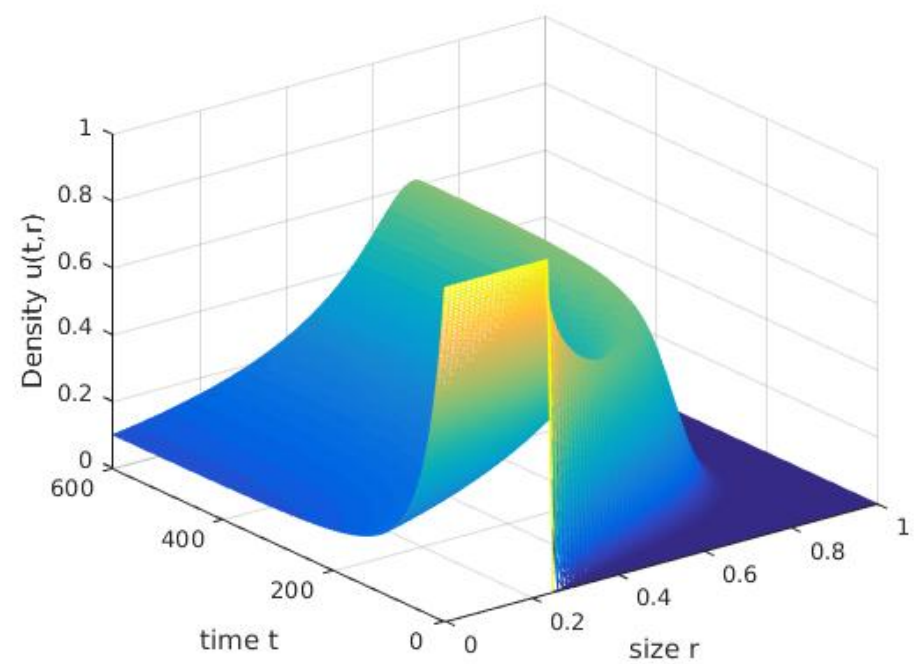

Figure 3: Simulation of the indirect transfer model with $\left(\alpha^{+}=0.75, \alpha^{-}=\right.$ $\alpha^{+} / 2$ and $\left.p(0)=0.6\right)$. The initial data for cells are given by (6.2). The initial concentration of the liquid is such that $\alpha^{+} p(0)>\alpha^{-}$. We observe a "right transfer" which means that the cells take up protein from the surrounding environmental liquid to increase their total quantity of protein.

\subsection{Experiment 2}

In this experiment we present a numerical simulation corresponding to the case $q(0)<0$. In this part we use the indirect transfer model only (i.e. $\widehat{\tau}=0$ ). This experiment was run with initial data $u_{0}$ chosen as follows

$$
u(0, r)=\left\{\begin{array}{lll}
0 & \text { if } & 0 \leq r \leq 0.75 \\
1 & \text { if } & 0.75 \leq r \leq 1
\end{array}\right.
$$

This initial distribution $u_{0}$ illustrates a density of resistant cells in a dish. Those cells have a larger level of P-gp expression. This means that the quantity of P-gp $r$ for resistant cells will vary in between 0.75 and 1 at time $t=0$. Moreover the surrounding liquid contains a quantity of protein given by $p(0)=$ 0.4 at time $t=0$.

Since $q_{0}=\left(\alpha^{+} p_{0}-\alpha^{-}\right)<0$, the map $q(t)=\left(\alpha^{+} p(t)-\alpha^{-}\right)$is a strictly negative function which is integrable. We observe a "left transfer" which means that the cells release protein into the surrounding environmental liquid to decrease their total quantity of protein. In figure (4) we can see that the successive 
curves converge to a steady curve. Moreover as time $t$ goes to infinity we have

$$
\begin{aligned}
Q(\infty) & :=\int_{0}^{+\infty} q(s) d s \in(-\infty, 0), \\
\lim _{t \rightarrow \infty} p(t) & =\frac{\alpha^{-}}{\alpha^{+}} \text {and } \\
\lim _{t \rightarrow+\infty} u(t) & =T_{A}(Q(\infty)) u_{0} .
\end{aligned}
$$

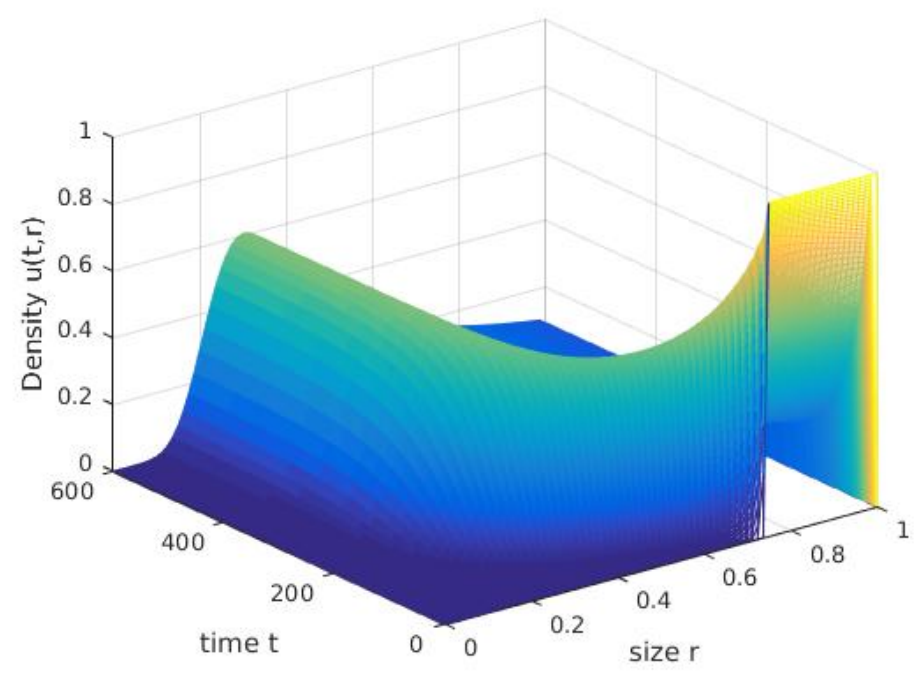

Figure 4: Simulation of the indirect transfer model with $\left(\alpha^{+}=0.75, \alpha^{-}=\right.$ $\alpha^{+} / 2$ and $\left.p(0)=0.4\right)$. The initial data for cells are given by (6.3). The initial concentration of protein in the liquid is such that $\alpha^{+} p(0)<\alpha^{-}$. We observe that the cells release protein into the surrounding environmental liquid to decrease their total quantity of protein.

\subsection{Experiment 3}

Both direct and indirect transfer (i.e. with $\widehat{\tau}>0$ ) with $q(0)>0$. The system constitutes two cell populations, sensitive cells and resistant cells. The initial data $u_{0}$ was chosen as follows

$$
u(0, r)=\left\{\begin{array}{lll}
1 & \text { if } & 0 \leq r \leq 0.25 \text { or } 0.75 \leq r \leq 1 \\
0 & \text { elsewhere }
\end{array}\right.
$$

This means there are sensitive cells corresponding to a lower quantity of protein (i.e. with $r$ in between 0 and 0.25 ), and resistant cells corresponding to higher 
quantity of protein (i.e. with $r$ in between 0.75 and 1 ). To simulate the indirect transfer, we chose $\eta($.$) as the following well adapted function$

$$
\eta(r)=r(1-r),
$$

and parameter values $\alpha^{+}=0.75$, and $\alpha^{-}=\alpha^{+} / 2$.

(a)

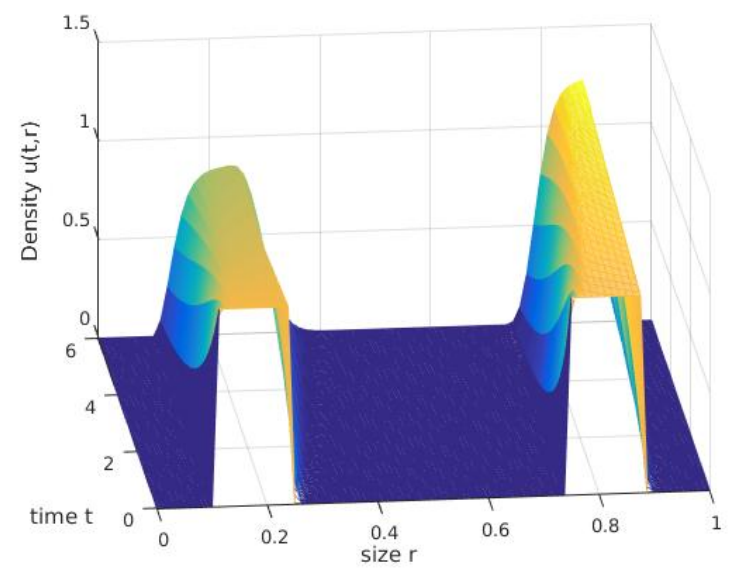

(b)

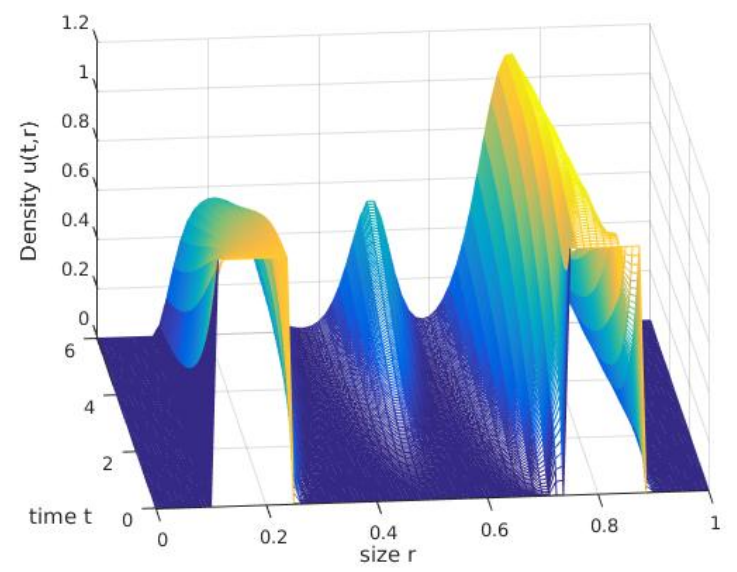

Figure 5: Direct and indirect transfer with $\alpha^{+} p(0)>\alpha^{-}$. In figure (a) we use the model with indirect transfer only, while in figure (b) we use a combination of direct and indirect transfer. The initial data for cells are given in (6.6). The initial concentration of the liquid is such that $\alpha^{+} p(0)>\alpha^{-},\left(\alpha^{+}=0.75, \alpha^{-}=\right.$ $\alpha^{+} / 2$ and $\left.p(0)=0.6\right)$. The time unit is one day, and we run the simulation from day 0 to day 6 . We observe that the cells take up proteins from the surrounding environmental liquid to increase their total quantity of protein. 


\subsection{Experiment 4: Simulations with experimental data}

In this simulation we calibrate and validate our model using experimental data taken from the paper [13]. We will see that the model with indirect and direct transfer gives a better fit to the experimental data than the model with only direct transfer.

Briefly, we recall the experiment described by the authors of [13]. The cell lines used were wild-type drug sensitive human breast adenocarcinoma MCF7 and a multi-drug resistant MCF-7 $\backslash$ DOXO variant. The cells were grown in dishes. Co-culture experiments were used to follow cell membrane P-gp contents over time, from day 0 to day 6 .

After $0,3,4,5$ and 6 days of co-culture a sample of 10000 cells was analyzed. Experimental data obtained were expressed in fluorescence units (f.u). According to the fluorescence $(\mathrm{F})$ scale used in cytometry, $F$ varies between $F_{\min }=1$ and $F_{\max }=10^{4}$. The fluorescence units were rescaled into an arbitrary Pglycoproteins unit by introducing a non-linear bijection $F:[0,1] \rightarrow\left[1,10^{4}\right]$ defined by $F(r)=10^{4 r}$ where $r$ denotes the P-glycoproteins expressed on the cell surface and $F$ its corresponding fluorescence value.

To calibrate the model and to estimate the parameters from given experimental data and observed results, we use the standard error ( $R M S E)$ of simulated

and observed data. Recall that $R M S E:=\sqrt{\sum_{l=1}^{N}\left(o b s\left(t_{l}\right)-\operatorname{sim}\left(t_{l}\right)\right)^{2} / N}$ where $N$ is the number of observed data, obs $\left(t_{l}\right)$ and $\operatorname{sim}\left(t_{l}\right)$ are the observed and simulated data at time $t_{l}$ respectively. In Table 2 we give the list of the parameters for this experiment. 


\begin{tabular}{|c|c|c|c|c|}
\hline Symbol & Description & Values & Units & \\
\hline$\overline{\bar{\tau}}$ & Rate of transfer of P-gp activity & 0.95 & $\mathrm{day}^{-1}$ & \\
\hline$F_{\min }$ & Minimum value of P-gp activity & 1 & f.u & data \\
\hline$F_{\max }$ & Maximum value of $\mathrm{P}$-gp activity & $10^{4}$ & f.u & \\
\hline$\delta_{\min }$ & Minimum threshold for transfers & $10^{4 * 0.41}$ & f.u & \\
\hline$\delta_{\max }$ & Maximum threshold for transfers & $10^{4 * 0.46}$ & f.u & \\
\hline $\bar{f}_{1}$ & Transfer efficiency for $\mathrm{p}$ value in $J$ & 0.48 & - & \\
\hline $\bar{f}_{2}$ & Transfer efficiency for $\mathrm{p}$ value in $J$ & 0.07 & - & \\
\hline$\epsilon \bar{f}_{1}$ & Transfer efficiency for $\mathrm{p}$ value in $J^{c}$ & 0.05 & - & \\
\hline $\begin{array}{l}\epsilon \bar{f}_{2} \\
\eta(.)\end{array}$ & $\begin{array}{l}\text { Transfer efficiency for } \mathrm{p} \text { value in } J^{c} \\
\eta_{1}(r)=r(1-r)\end{array}$ & 0.007 & - & \\
\hline$\alpha^{+}$ & & 0.75 & & \\
\hline$\alpha^{-}$ & & $\alpha^{+} / 2$ & & \\
\hline
\end{tabular}

Table 2: List of the model parameters, their descriptions, values and symbols for Experiment 4. The model is simulated with an arbitrary P-glycoproteins unit $r \in[0,1]$ and by using a rescaling argument $F(r):=10^{4 r} \in\left[1,10^{4}\right]$ one gets its equivalent in terms of fluorescence units (f.u). Here $J:=\left(\delta_{\min }, \delta_{\max }\right)$ and $J^{c}$ its complement.

In Figure 6 we plot the data and the solution of our model obtained for the best fit of the parameters. 

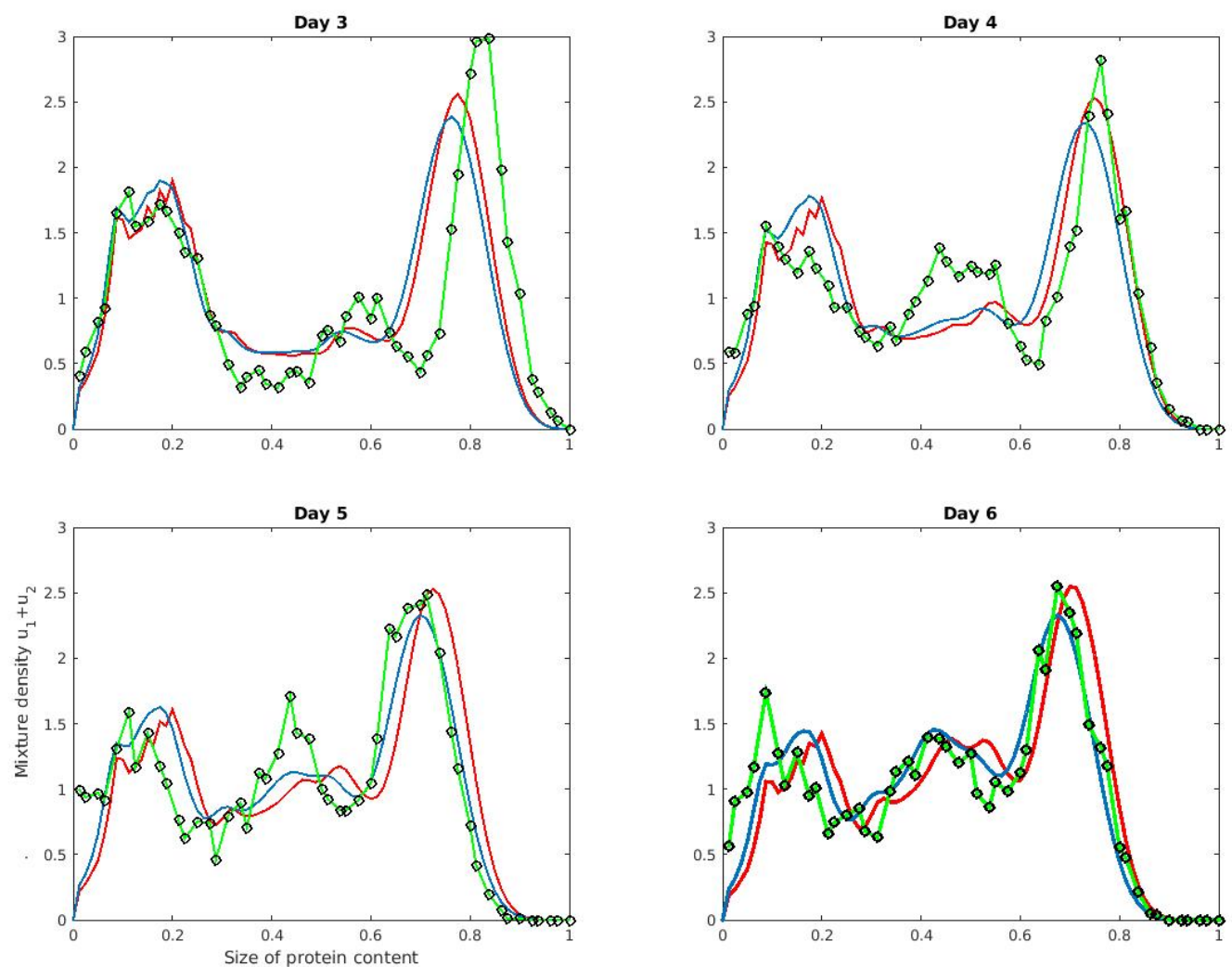

Figure 6: Comparison between direct (red curve), indirect and direct transfer (blue curve) and the data (green curve), at the days 3, 4, 5 and 6. The inclusion of the quantity of protein released in the liquid and transferred to the cells, via the indirect transfers, gives a better fit to the experimental data.

In Figure 7 we plot the $R M S E$ between the data and the solution of our model obtained for the best fit of the parameters. 


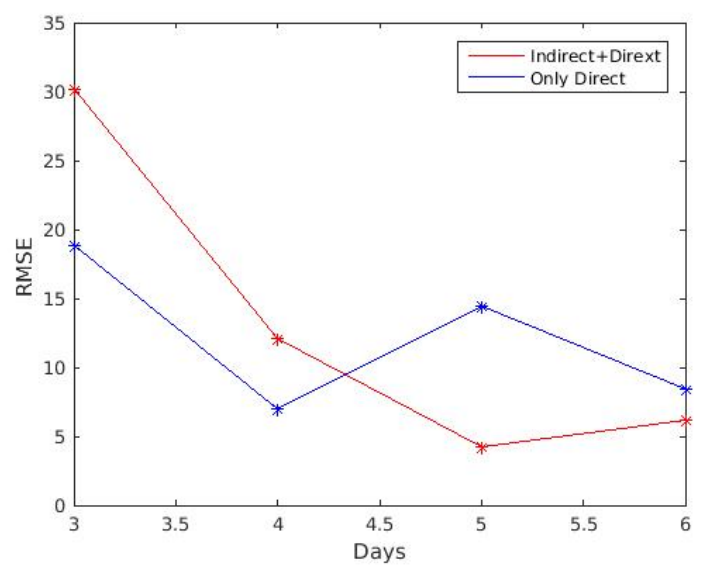

Figure 7: We report the Standard Root Mean Square Error RMSE between simulations and the observed data. We use the data at days 3, 4, 5 to 6.

\section{Conclusion and discussion}

Since P-glycoprotein is the main efflux pump for many chemotherapeutic agents, genomic and non-genomic P-gp acquisition are of prime importance in the biology of resistance to anticancer drugs within growing tumours. Despite two decades of intense pharmacological efforts to develop P-gp blockers, also known as chemosensitizers, no multidrug resistance reversal treatment is currently available on the market. Most of candidates did not pass Phase II trials of the clinical phase in drug development. Anyway, P-gp inhibition often causes unpredictable and dangerous side effects (see for example Chung et al. [3], ElAwady et al. [5]). Pharmacologic inhibition of efflux pumps remains challenging due to limits in balancing toxicity risks against therapeutic gains.

Therefore, a precise understanding of drug resistance development in time and space gives valuable information to manage cytotoxic anticancer treatments and to determine the best trade-off window in terms of dose and pharmacokinetic.

In this regard, the dynamics of P-gp dissemination by cell-cell transfers has obviously been underestimated and is not taken into account in chemotherapy protocols. Quantitative mathematical models based on biological observation are therefore of prime importance in evaluating the contribution of transfers to the overall drug resistance of tumours and, potentially, to help to determine optimized time-courses for chemotherapy.

In this paper, a model was formulated to describe the direct cell-to-cell transfers and the indirect transfers with the surrounding environment. Cell populations were structured by a continuous variable corresponding to transferable material while the time was considered to be continuous. The model extends the 
previous model developed in $[6,13]$, by introducing different types of efficiency transfer rates and by adding the indirect transfers with the liquid surrounding the cells. The preservation of the total number of cells, and the preservation of transfer mass quantity is guaranteed. Moreover a discrete version of the model which preserves the above conservation laws was also presented.

The model was able to reproduce experimental data obtained from experiments on the consequences of cell-to-cell protein transfer in breast cancer. Other choices of the function $\eta$ could have been used. This question is left for future investigations.

The computations using our algorithm yield quite good results. It is our opinion that this is due in large measure to the fact that the discrete approximating solution has the same long-term dynamics as the underlying continuous system.

In Experiment 4, we observe that the protein in the liquid may influence (in a complex manner) the results. This quantity of protein is usually neglected by the experimenters. By comparing the $R M S E$ values for both scenarios, one can conclude that taking into account both direct and indirect transfers gives a good fit of data compared to the case with only direct transfers.

Our work is motivated by a particular application demonstrating the direct cell- cell transfer and the indirect transfer of the surface protein P-glycoprotein (P-gp) present in cancer cells [13]. The significance of direct cell-cell transfer of P-gp has potential importance in understanding the development of resistance to cancer drugs, since P-gp is a cellular efflux pump for many chemotherapeutic agents.

\section{Appendix 1: The numerical scheme}

Selecting a mesh size $\Delta r>0$, a time step $\Delta t>0$ and a final time $T>0$ we show how to obtain the approximate solution in the interval $[0, T] \times[0,1]$. We use the framework of the finite volume method (see Leveque [8] for more results this topic) and we define a generalized upwind scheme. We set for any $i=1, \ldots, I, r_{1 / 2}=0$ and $r_{i+1 / 2}=r_{1 / 2}+i \Delta r$ where I is the integer part of $1 / \Delta r$.

We define the first mesh cell $\left.M_{1}^{\Delta}=\right] 0, r_{1+1 / 2}$ [ with the middle point $r_{1}=$ $\Delta r / 2$, and the last mesh cell $\left.M_{I}^{\Delta}=\right] r_{I-1 / 2}, 1\left[\right.$ with the middle point $r_{I}=$ $\left(r_{I-1 / 2}+1\right) / 2$. We define the meshes $\left.M_{i}^{\Delta}=\right] r_{i-1 / 2}, r_{i+1 / 2}[$ with the middle point $r_{i}=r_{i-1 / 2}+\Delta r / 2$ for any $i=1, \ldots,(I-1)$. In this way the interval $[0,1]$ is discretized with the cells $M_{i}^{\Delta}$. We introduce the sequence $t_{n}=n \Delta t$. At the initial time, corresponding to $\mathrm{n}=0$, we define $u^{0}=\left(u_{1}^{0}, \ldots, u_{I}^{0}\right)$ and $p^{0}=p(0)$. We suppose that $u^{n}=\left(u_{1}^{n}, \ldots, u_{I}^{n}\right)$ and $p^{n}$ have been constructed for $t \leq t_{n}$, where $u_{i}^{n}$ is an approximation of the exact value of $u\left(t_{n}, i \Delta r\right)$. Assume the Courant-Friedrich-Lewy (CFL) restriction holds

$$
\frac{\Delta t}{\Delta r} \sup _{n, i}\left|p^{n} \eta\left(r_{i+1 / 2}\right)\right|<1,
$$

We define the fluxes at boundary interfaces of the meshes $\left.M_{i}^{\Delta}=\right] r_{i-1 / 2}, r_{i+1 / 2}[$ 
by

$$
\eta_{i-1 / 2} u_{i-1 / 2}^{n}= \begin{cases}\eta\left(r_{i-1 / 2}\right) u_{i-1}^{n}, & \text { if } \alpha^{+} p^{n}>\alpha^{-} \text {or } i=1, \ldots, I \\ \eta\left(r_{i-1 / 2}\right) u_{i}^{n} & \text { otherwise. }\end{cases}
$$

Now $p($.$) is approximated by a piecewise constant function p^{\Delta}($.$) given by$ $p^{\Delta}(t)=p^{n}$, for $t \in\left[t_{n}, t_{n+1}\right.$ [, where $p^{n}$ is the value generated by an Euler or Runge-Kutta scheme for example.

We obtain the following scheme for the density

$$
\begin{aligned}
u_{i}^{n+1}=u_{i}^{n} & -\frac{\Delta t}{\Delta r}\left[\alpha^{+} p^{n}-\alpha^{-}\right]\left[\eta_{i+1 / 2} u_{i+1 / 2}^{n}-\eta_{i-1 / 2} u_{i-1 / 2}^{n}\right] \\
& \left.+2 \widehat{\tau} \Delta t\left(T_{i}^{n}\left(u^{n}\right)-u_{i}^{n}\right)\right)
\end{aligned}
$$

and

$$
p^{n+1}=p^{n}+\Delta t\left(\alpha^{+} p^{n}-\alpha^{-}\right) \sum_{i=1}^{I} \eta_{i} u_{i}^{n} \Delta r .
$$

where $i$ varies from 1 to $I$ in (8.3), and we assume a null flux condition at $r=0$ and $r=1$ : that is $\eta_{1 / 2} u_{1 / 2}^{n}=0$ and $\eta_{I+1 / 2} u_{I+1 / 2}^{n}=0$.

This scheme works under the CFL condition (8.1). Here the transfer operator is approximated by

$$
\begin{aligned}
T_{i}^{n}\left(u^{n}\right) & =\frac{1}{\left|u^{n}\right|_{1, \Delta}} \sum_{j \in \mathbb{Z}} \pi\left(r_{i}\right) \bar{u}_{i-f_{1} j}^{n} \bar{u}_{i+\left(1-f_{1}\right) j}^{n} \Delta r \\
& +\frac{1}{\left|u^{n}\right|_{1, \Delta}} \sum_{j \in \mathbb{Z}}\left(1-\pi\left(r_{i}\right)\right) \bar{u}_{i-\left(1-f_{2}\right) j}^{n} \bar{u}_{i+f_{2} j}^{n} \Delta r
\end{aligned}
$$

where the quantities $i-f_{1} j, i+\left(1-f_{1}\right) j, i-f_{2} j$ and $i+\left(1-f_{2}\right) j$ are understood respectively as their integer part in the system (8.5).These above indexes correspond to the partner cells which interact directly. Here $\left|u^{n}\right|_{1, \Delta}$ corresponds to the discrete $L^{1}$-norm

$$
\left|u^{n}\right|_{1, \Delta}=\sum_{i=1}^{I}\left|u_{i}^{n}\right| \Delta r
$$

and

$$
\bar{u}_{i}:=\left\{\begin{array}{l}
u_{i} \text { if } i=1, \ldots, I, \\
0 \text { otherwise. }
\end{array}\right.
$$

We can see that the total number of cells is conserved

$$
\sum_{i=1}^{I} u_{i}^{n} \Delta r=\sum_{i=1}^{I} u_{i}^{0} \Delta r
$$

and the total mass of transferable quantities is preserved in time

$$
p^{n}+\sum_{i=1}^{I} r_{i} u_{i}^{n} \Delta r=p^{0}+\sum_{i=1}^{I} r_{i} u_{i}^{0} \Delta r .
$$




\section{Acknowledgement}

The authors would like to thank the anonymous reviewers for their valuable comments and suggestions to improve the quality of the paper.

\section{References}

[1] S. V. Ambudkar, Z.E. Sauna, M. M. Gottesman, and G. Szakacs, A novel way to spread drug resistance in tumor cells: Functional intercellular transfer of P-glycoprotein (ABCB1), Trends Pharmacol. Sci., 26 (2005), 385387.

[2] M. L. Amin, P-Glycoprotein Inhibition for Optimal Drug Delivery, Drug Target Insights 7 (2013), 27-34.

[3] F.S. Chung et al. , Disrupting P-Glycoprotein Function in Clinical Settings: What Can We Learn from the Fundamental Aspects of This Transporter?, American Journal of Cancer Research 6(8) (2016), 1583-1598. Print.

[4] C. D'Souza-Schorey and J. W. Clancy, Tumor-derived microvesicles: shedding light on novel microenvironment modulators and prospective cancer biomarkers. Genes \& development 26 (2012), 1287-1299.

[5] R. El-Awady, E. Saleh, A. Hashim, N. Soliman, A. Dallah, A. Elrasheed, G. Elakraa, The Role of Eukaryotic and Prokaryotic ABC Transporter Family in Failure of Chemotherapy, Front. Pharmacol. 7 (2016), 535.

[6] P. Hinow, F. Le Foll, P. Magal, G. F. Webb, Analysis of a model for transfer phenomena in biological populations, SIAM J. Appl. Math. 70 (2009), 4062 .

[7] T. Kato, Perturbation theory for linear operators, Springer-Verlag, 1976.

[8] R. J. Leveque Finite Volume Methods for Hyperbolic Problems, Cambridge University Press (2002).

[9] A. Levchenko, B. M. Mehta, X. Niu, G. Kang, L. Villafania, D. Way, D. Polycarpe, M. Sadelain, and S. M. Larson, Intercellular transfer of Pglycoprotein mediates acquired multidrug resistance in tumor cells, Proc. Natl. Acad. Sci. USA 102 (2005), 1933-1938.

[10] V. Lopes-Rodrigues et al., Identification of the metabolic alterations associated with the multidrug resistant phenotype in cancer and their intercellular transfer mediated by extracellular vesicles, Scientific reports 7 (2017), 44541 .

[11] P. Magal, A. Noussair, J. Pasquier, P. Zongo and F. Le Foll, A model for transfer of P-glycoproteins in MCF-7 breast cancer cell line with multiple transfer rules, Bull. Math. Biol. 79 (2017), 2049-2067. 
[12] J. Pasquier, P. Magal, C. Boulangé-Lecomte, G. F. Webb, and F. Le Foll, Consequences of cell to cell $\mathrm{P}$ - glycoprotein transfer on acquired multidrug resistance in tumors: A cell population dynamics model, Biology Direct 6 (2011). http://www.biology-direct.com/content/6/1/5.

[13] J. Pasquier, L. Galas, C. Boulange-Lecomte, D. Rioult, F. Bultelle, P. Magal, G. F. Webb and F. Le Foll, Different modalities of intercellular membrane exchanges mediate cell-to-cell P-glycoprotein transfers in MCF7 breast cancer cells, Journal of Biological Chemistry 287(10) (2012), 7374-7387.

[14] A. Pazy, Semigroups of linear operators and applications to partial differential equations. Springer-Verlag, Berlin 1983.

[15] P. Samuel, M Fabbri, D. Carter. Mechanisms of Drug Resistance in Cancer: The Role of Extracellular Vesicles, Proteomics 17 (2017).

[16] P. Samuel, L.A. Mulcahy, F. Furlong et al. Cisplatin induces the release of extracellular vesicles from ovarian cancer cells that can induce invasiveness and drug resistance in bystander cells, Phil. Trans. R. Soc. B 373 (2018), 20170065.

[17] L. Sivak, V. Subr, J. Tomala, B. Rihova, J. Strohalm, T. Etrych and M. Kovar, Overcoming multidrug resistance via simultaneous delivery of cytostatic drug and P-glycoprotein inhibitor to cancer cells by HPMA copolymer conjugate, Biomaterials 115 (2017), 65-80.

[18] G. F. Webb, Population models structured by age, size, and spatial position. (Editors P. Magal and S. Ruan) In Structured population models in biology and epidemiology (pp. 1-49). Springer Berlin Heidelberg (2008). 